\title{
Multiplexing holograms for data page storage as a holographic memory in a PVA/AA photopolymer
}

\author{
Elena Fernández ${ }^{* a}$, Manuel Ortuño ${ }^{b}$, Sergi Gallego ${ }^{\mathrm{b}}$, Celia García ${ }^{\mathrm{a}}$, Andrés Márquez ${ }^{\mathrm{b}}$, Augusto \\ Beléndez $^{\mathrm{b}}$ and Inmaculada Pascual ${ }^{\mathrm{a}}$ \\ ${ }^{a}$ Dept. Óptica, Universidad de Alicante, Apartado 99, E-03080 Alicante, Spain \\ ${ }^{\mathrm{b}}$ Dept. de Física, Ing. de Sistemas y T $\mathrm{T}^{\mathrm{a}}$ de la Señal, Universidad de Alicante, Ap. 99, E-03080 \\ Alicante, Spain
}

\begin{abstract}
Holographic data pages were multiplexed in a PVA/acrylamide photopolymer layer. This material is formed of acrylamide photopolymers which are considered interesting materials for recording holographic memories. A liquid crystal device was used to modify the object beam and store the data pages in the material. Objects with different pixel size have been used to simulate the data pages. A peristrophic multiplexing method is used to store a large number of data pages in the same spot in the material. The Bit Error Rate (BER) was calculated fitting the histograms of the images to determine the quality of the images.
\end{abstract}

Keywords: Photopolymers, Holographic recording materials, Liquid crystal device.

\section{INTRODUCTION}

Due to the importance acquired by new technologies (computers and Internet), the demand for more capacity, more density and faster readout rates has increased considerably. The conventional optical memory technologies, like CDROM's and DVD's, are two-dimensional surface-storage techniques, and so have almost arrived at the limit of their capacity. For this reason, in recent years a lot of attention has been centred on 3-dimensional holographic disks ${ }^{1-3}$. Recently, many studies have been focused on the characterisation and optimization of thick holographic recording materials in order to obtain the maximum data storage capacity.

Since photopolymers have excellent holographic characteristics, such as high refractive index modulation ${ }^{4,5}$, large dynamic range ${ }^{6-8}$, good light sensitivity, real time image development, high optical quality and low cost, they have been used as the base of new 3-D holographic disks. In addition to this, their properties like energetic sensitivity or spectral sensitivity can be easily changed by modifying their composition ${ }^{4,6,9}$.

In this study, we focus on the optimization of a holographic memory setup multiplexing a large number of data pages using a liquid crystal display (LCD) and a PVA-acrylamide photopolymer.

Twisted-nematic liquid crystal displays (TN-LCDs) have been extensively studied in recent years for application to spatial light modulators (SLMs) used in many applications in optics to modify in real time the amplitude or phase of a light beam ${ }^{10-14}$. This LCD can be used to design programmable optical elements, such as lenses, data pages or in holographic data storage. In particular in holographic data storage, LCDs allow data pages to be recorded in real time in the holographic recording material.

The photopolymer is composed of acrylamide (AA) as the polymerizable monomer, triethanolamine (TEA) as radical generator, N,N'methylene-bis-acrylamide (BMA) as crosslinker, yellowish eosin (YE) as sensitizer and a binder of polyvinyl alcohol (PVA) ${ }^{4,9}$. The layers are about $80 \pm 10 \mu \mathrm{m}$ thick.

Different objects with white and black pixels were used to simulate data page bits (ones and zeros). During the storage process, these data pages are stored in the photopolymer. When the hologram has been stored, it is illuminated in the reconstruction stage by the same plane wave as in the recording process in order to prevent the appearance of

Practical Holography XXII: Materials and Applications, edited by Hans I. Bjelkhagen, Raymond K. Kostuk, Proc. of SPIE Vol. 6912, 691214, (2008) · 0277-786X/08/\$18 - doi: 10.1117/12.762565 
aberrations in the reconstructed image. Using an optical system, the stored information is imaged onto a Cohu 4710 Series Monochrome CCD camera connected to a personal computer, where the images are analyzed and processed.

Once the images have been obtained, a criterion has to be used to assess the quality of the different images and to compare them with the original object. In order to evaluate the image quality, its histogram is used to calculate the Bit Error Rate (BER) ${ }^{15-17}$ and determine the contrast between white and black pixels. BER values of each image are calculated to decide what parameters provide the best image quality (greater contrast and less noise).

\section{EXPERIMENTAL}

\subsection{Preparation of the material}

The holograms are registered in a photopolymer composed of acrylamide (AA) as the polymerizable monomer, triethanolamine (TEA) as radical generator, N,N'methylene-bis-acrylamide (BMA) as crosslinker, yellowish eosin (YE) as sensitizer and a binder of polyvinyl alcohol (PVA). Introduction of BMA in the composition improves the energetic sensitivity and diffraction efficiency of the material and, in addition, gives a greater stability to the stored grating, thereby preventing it from disappearing with time.

Table 1 shows the component concentrations of the photopolymer composition used to obtain layers about $80 \mu \mathrm{m}$ thick.

Table 1: Concentrations of the photopolymer composition.

\begin{tabular}{cc}
\hline & Composition \\
\hline Polyvinylalcohol & $6.6 \% \mathrm{w} / \mathrm{v}$ \\
Acrylamide & $0.33 \mathrm{M}$ \\
Triethanolamine & $0.17 \mathrm{M}$ \\
Yellowish eosin & $2.4 \cdot 10^{-4} \mathrm{M}$ \\
N,N'methylene-bis-acrylamide & $0.027 \mathrm{M}$ \\
\hline
\end{tabular}

A solution of PVA in water forms the matrix and this is used to prepare the mixture of AA, BMA, and photopolymerization initiator system composed of TEA and YE. The mixture is made under red light, deposited by gravity on a $22 \mathrm{~cm} \times 40 \mathrm{~cm}$ glass plate and left in the dark for one day to allow the water to evaporate in conditions of temperature, $\mathrm{T}$, between $20^{\circ} \mathrm{C}$ and $25{ }^{\circ} \mathrm{C}$, and relative humidity, $\mathrm{RH}, 40 \%-60 \%$. These conditions of drying time, temperature and relative humidity are optimized to obtain the maximum diffraction efficiency of the gratings. Once dry, the glass is cut into squares of $5 \mathrm{~cm} \times 5 \mathrm{~cm}$.

\subsection{Holographic set-up}

Holographic data pages were recorded using the output from a diode-pumped frequency-doubled $\mathrm{Nd} \mathrm{YVO}_{4}$ laser (Coherent Verdi V2) which was split into two beams and then spatially filtered, using a microscope objective lens and a pinhole, and collimated to yield a plane-wave source of light at $532 \mathrm{~nm}$. The diameters of these beams were $1.5 \mathrm{~cm}$. The two laser beams were spatially overlapped at the recording medium intersection at an angle of $17.4^{\circ}$ (measured in air).

One of the beams was the object beam and the other was the reference beam. In the object beam the LCD was placed between two polarizers, one to each side of the LCD. The set of LCD and polarizers were used like a SLM. In addition, a lens in front of the SLM was placed to do the Fourier transform (FT) of the data page which was sent to the SLM. Just in front of the photopolymer a diaphragm was placed to block all the orders that leave the LCD except the central order. If the other orders were not blocked, they would also be stored in the material, and during reconstruction interference patterns would be observed on the image, thus worsening its quality. The other beam, the reference beam, was a plane wave that interferes with the object beam at the surface of the material. In this work, we studied how the beam ratio between object beam and reference beam affects the quality of the stored images. These beam intensities were measured at the position where the photopolymer must be placed when the holograms are stored. In the reconstruction stage, the 
stored hologram that contains the information of the data page was illuminated with the reference beam, but with a very low intensity so as not to deform the hologram, because the material is sensitive to this wavelength. Another lens was placed behind the photopolymer to do the inverse Fourier transform (IFT) of the diffracted beam on the surface of the charge coupled device (CCD). A computer sends the data pages to the LCD and captures images reconstructed by the CCD.

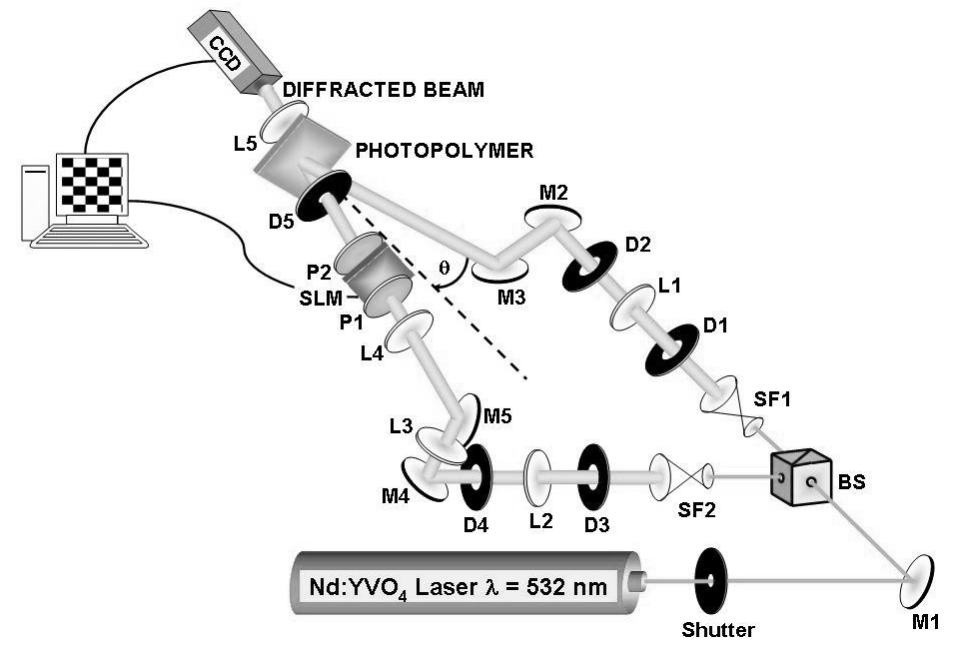

Figure 1: Experimental setup: BS beam splitter, Mi mirror, Li lens, Di, diaphragm, SFi, microscope objective lens and pinhole, SLM spatial light modulator, Pi polarizer, CCD charge coupled device.

\section{RESULTS}

In this paper holograms have been multiplexed in layers about $80 \mu \mathrm{m}$ using the composition in table 1. Binary data pages with a random pixel form were stored. These objects have a different number of pixels: 300x300, 400x400, $500 \times 500$ and $800 \times 600$ pixels. This allows us to study the behaviour of the photopolymer material when data pages with different numbers of pixels are multiplexed.

These objects were stored in the same position in the material using peristrophic multiplexing because previous papers showed that when a not very large number of holograms (fewer than 200 holograms) are to be stored, higher diffraction efficiency is obtained with peristrophic multiplexing ${ }^{18}$.

The holograms were stored with an angular separation of $3^{\circ}$. The angular selectivity of the layers was measured in this study. For a thickness of $80 \mu \mathrm{m}$, we obtained an angular selectivity of $2^{\circ}$. Therefore, with an angular separation of $3^{\circ}$ is more than sufficient to prevent the holograms from overlapping.

The holograms were stored with a reference to object beam ratio of 100 and a reading beam intensity of $0.03 \mathrm{~mW} / \mathrm{cm}^{2}$. In a previous study on the influence of these parameters on the quality of the stored images ${ }^{19}$, these values were found to give a greater image quality and, therefore, a lower BER.

\subsection{Optimization of the LCD}

As described in the Holographic set-up section, an LCD was placed in the object beam to modify the wavefront and store this variation in the photopolymer. The variation may be in phase or amplitude. In this study, the wavefront amplitude is modified while its phase is maintained constant, and this modification is the object to be stored in the photopolymer.

LCDs change the polarization state of the incident light. This may mean that not all the light incident on the LCD is transmitted, since some of it is reflected or absorbed. Therefore, it is advisable to place two polarizers, one before and one after the LCD, in order to transmit the maximum light possible. However, to achieve this, the LCD and polarizers must be calibrated correctly, which means that the angles at which the latter are to be placed in order to achieve maximum efficiency and minimum loss must be calculated. In our study, the objective was also to obtain the maximum contrast between transparent and opaque zones of the LCD with no or only minimum phase variation. Therefore, before 
sending the objects to the LCD for storage in the photopolymer, the LCD must be correctly calibrated.

This is done using the method described in references ${ }^{12,20}$, which consists of two calibration steps. In the first step, the LCD is turned off and no voltage is applied. In this step, three parameters that are independent of the voltage are calculated: the total twist angle $(\alpha)$, the orientation of the molecular director at the input face $\left(\Psi_{\mathrm{D}}\right)$ and the maximum birefringence $\left(\beta_{\max }\right)$. The parameters obtained with the LCD calibrated while turned off are shown in table 2 .

Table 2: LCD Calibration parameters independent of the voltage applied.

\begin{tabular}{ccccc}
\hline$\alpha$ & $\Psi_{\mathrm{D}}$ & $\beta_{\max }\left(\lambda_{0}=633 \mathrm{~nm}\right)$ & $\beta_{\max }\left(\lambda_{0}=532 \mathrm{~nm}\right)$ & $\beta_{\max }\left(\lambda_{0}=442 \mathrm{~nm}\right)$ \\
\hline $94^{\mathrm{o}} \pm 1^{\mathrm{o}}$ & $45^{\circ} \pm 1^{\mathrm{o}}$ & $118^{\mathrm{o}} \pm 1^{\mathrm{o}}$ & $168^{\circ} \pm 1^{\mathrm{o}}$ & $199^{\circ} \pm 1^{\mathrm{o}}$ \\
\hline
\end{tabular}

In the second step, the parameters dependent on voltage are measured. These are related to the variation in optical anisotropic properties throughout the thickness of the cell as a function of the voltage applied. The model attempts to take into account the fact that the liquid crystal molecules near the glass are practically adhered to its surface and cannot reorientate themselves when the voltage is applied. Thus, the total thickness $\mathrm{d}$ of the LCD may be decomposed into two lateral regions of width $d_{1}$ and a central region of width $d_{2}$. In this way, the anisotropic properties of the LCD may be modeled using two voltage-dependent parameters - birefringence $\beta$ and $\delta$-which are expressed in Eq. 1 .

$$
\begin{aligned}
& \beta(V)=\pi \Delta n d_{2} / \lambda_{0} \\
& \delta(V)=\pi \Delta n_{\text {max }} d_{1} / \lambda_{0}
\end{aligned}
$$

where $\lambda_{0}$ is the wavelength of the light, $\Delta \mathrm{n}$ is the difference between the ordinary and extraordinary index, with $\Delta \mathrm{n}_{\max }$ being the maximum value.

From the curves of $\beta(\mathrm{V})$ and $\delta(\mathrm{V})$, we can find the angles at which the polarizers must be placed in the experimental setup to modulate the incident beam. In our study, our aim is to obtain the maximum contrast between transparent and opaque zones of the LCD with minimum variation in the phase. In this case, the angles found are $\varphi_{1}=296^{\circ}$ for the polarizer in front of the LCD and $\varphi_{2}=111^{\circ}$ for the polarizer behind it.

\subsection{Calculation of the Bit Error Rate}

After one of the objects has been stored in the photopolymer, the hologram formed is reconstructed illuminating it with the reference beam. The diffractive beam obtained is imaged onto the CCD. If the holographic reconstruction was perfect, the images obtained would have uniform white and black pixels. However many reasons may to distort the image. For this reason, it is convenient to measure a parameter that quantifies the image quality. This parameter is the Bit Error Rate (BER).

The BER is defined as the probability of having erroneous bits in the image. To calculate the BER ${ }^{15,19}$, first, in Fig. 2 , we represent, in logarithmic scale, the probability of obtaining a certain gray level in the black or in the white regions. In this figure, the probability in the black regions is represented by solid circles and the probability in the white regions is represented by empty circles. As can be seen, the two probability distributions are clearly distinguishable, although there is a point at which they intersect. This point of intersection of the two distributions is called $\mathrm{x}_{\mathrm{c}}$.

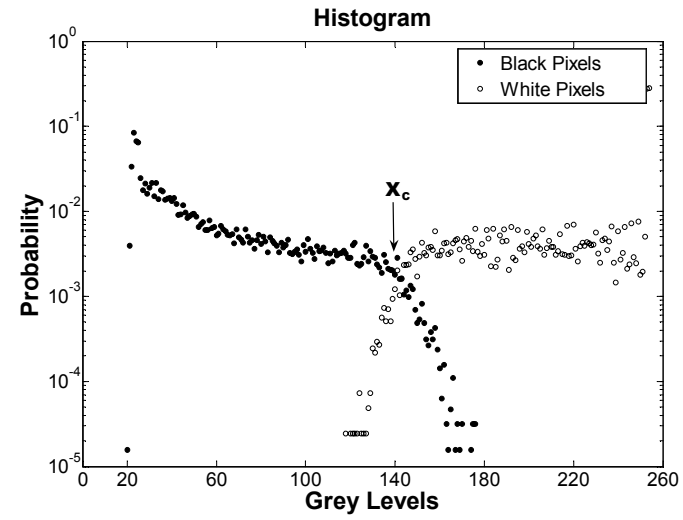

Fig. 2: Distribution of the probability of black and white pixels. 
Once the probability distributions have been obtained from the graph (Fig. 2), both distributions are fitted to a probability function. In this study, they are fitted to a Gaussian equation (Eq. 2).

$$
W\left(x_{0}, \sigma ; x\right)=\frac{1}{\sqrt{2 \pi \sigma}} \exp \left(-\frac{\left(x-x_{0}\right)^{2}}{2 \sigma^{2}}\right)
$$

where $\mathrm{x}$ represents each gray level in the image, $\mathrm{x}_{0}$ the point at which the Gaussian distribution is centered and $\sigma$ the width of the Gaussian distribution. The reason why they are fitted to a Gaussian equation is that it has been verified that most probability distributions obtained from an image captured by a CCD may be expected to follow this type of distribution.

Once the adjustments for the probability distribution of both white and black pixels have been made, the BER is calculated from Eq. 3:

$$
B E R=\frac{1}{2}\left[\int_{0}^{x_{c}} W_{W}(x) d x+\int_{x_{c}}^{\infty} W_{B}(x) d x\right]
$$

where $\mathrm{W}_{\mathrm{W}}$ and $\mathrm{W}_{\mathrm{B}}$ are the adjustments of the probability distribution of white and black pixels respectively and $\mathrm{x}_{\mathrm{c}}$ is the point of intersection of the two probability distributions.

Using this algorithm, the BERs of all the images were calculated and are discussed in the following sections.

\subsection{Thickness of $80 \mu \mathrm{m}$.}

As mentioned above, data pages were stored with four different pixel sizes. In this section, these four data pages are multiplexed in the $80 \mu \mathrm{m}$ thick material. Each of the four objects is stored at the same position in the material with an angular separation of $3^{\circ}$. In addition, they are stored with a beam ratio of 100 and reconstructed on the CCD with a reading beam intensity of $0.03 \mathrm{~mW} / \mathrm{cm}^{2}$. As the holograms are stored in the material, the dynamic range is consumed, ${ }^{15-}$ ${ }^{19}$; therefore, the exposure must be increased in order to store more holograms. Of course, when all the dynamic range is consumed, no more holograms will be formed in the material even if the exposure is increased.

Thus, when the 300x300 pixel object was stored, 15 holograms were multiplexed in the material. Fig. 3 shows the BER of the four multiplexed data pages as a function of the number of holograms. The BER obtained from each of the 15 holograms are represented by solid black circles. As can be seen, the first 9 holograms have a BER less than 0.1 and the holograms up to number 12, less than 0.2. However, the BER of the last holograms increases and reaches 0.4 for hologram 15. For the last holograms, no matter how much the exposure was increased, images were obtained with little contrast between pixels, thus indicating that this is the limit for storing images with this thickness and this number of pixels.

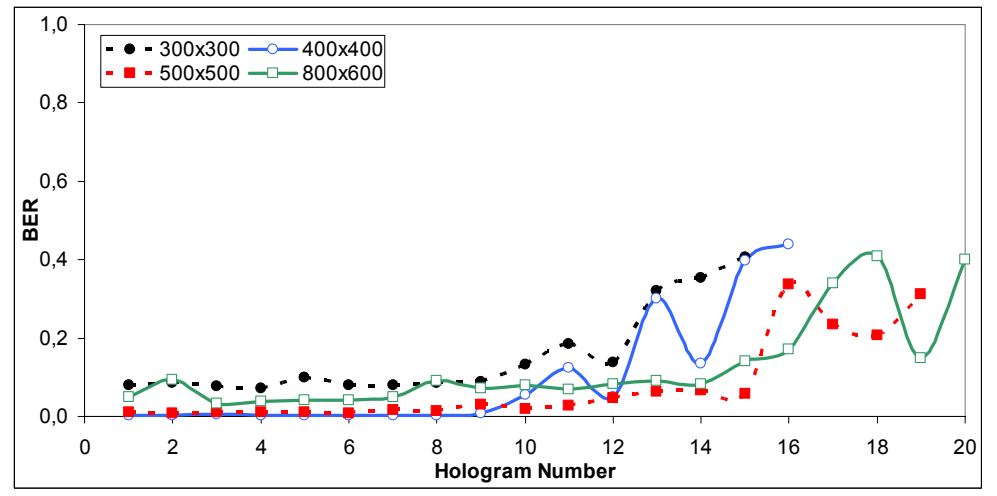

Fig. 3: BER of the objects of 300x300 pixels (solid black circles), 400x400 pixels (empty blue circles), 500x500 pixels (solid red squares) and 800x600 pixels (empty green squares) in a $80 \mu \mathrm{m}$ thick material.

Fig. $4 \mathrm{a}$ and $4 \mathrm{~b}$ show the images of the first and last holograms, reconstructed with the reference beam. In Fig. 4a there is a greater contrast between the white and black pixels and the edges are well-defined. However, in Fig. 4b although 
some areas are whiter than others, it is not possible to clearly distinguish the edges of the pixels, since the hologram was not well formed. This deterioration in the quality of the image is what makes the BER increase.

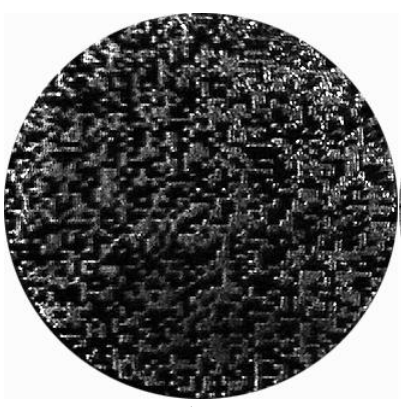

a)

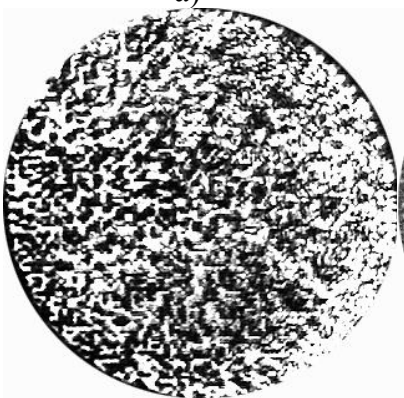

c)

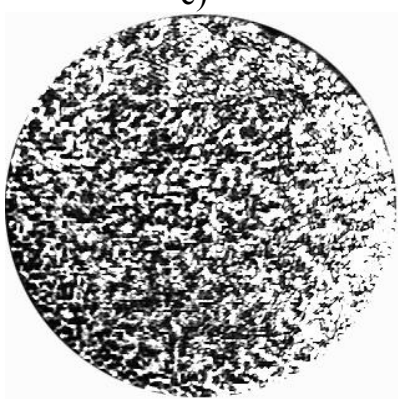

e)

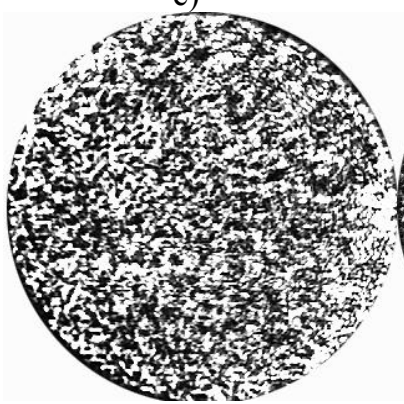

g)

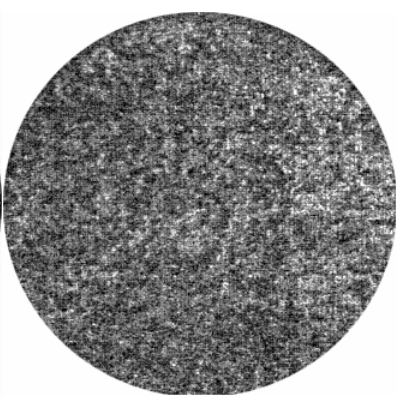

b)

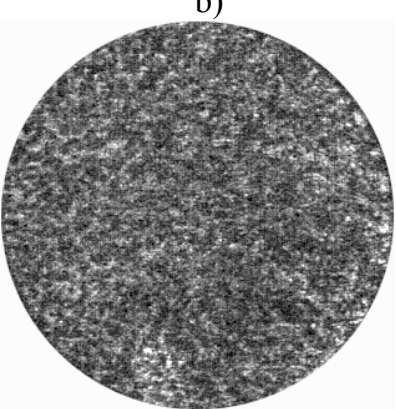

d)

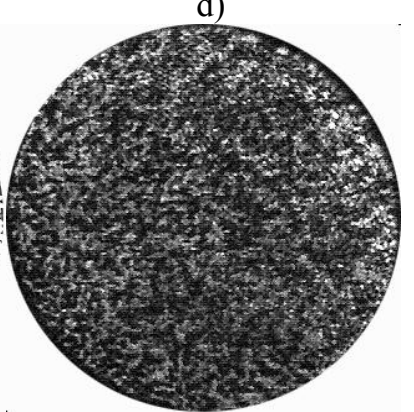

f)

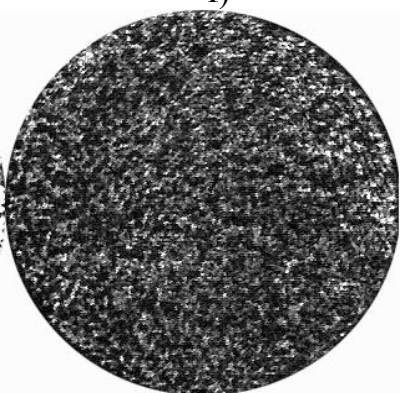

h)

Fig. 4: First and last images corresponding to objects with $300 \times 300$ pixels $(\mathrm{a}, \mathrm{b}), 400 \times 400$ pixels $(\mathrm{c}, \mathrm{d}), 500 \times 500$ pixels $(\mathrm{e}, \mathrm{f})$ and $800 \times 600$ pixels $(\mathrm{g}, \mathrm{h})$ in a $80 \mu \mathrm{m}$ thick layer.

In the following step, holograms with 400x400 pixels were multiplexed and 16 holograms were stored. In Fig. 3 the BER of these 16 holograms are represented by empty blue circles. As can be seen again, the first holograms have a BER close to 0 , whereas from hologram 13 on the BER begins to increase. Figs. $4 \mathrm{c}$ and $4 \mathrm{~d}$ show the first and last holograms of the $400 \times 400$ pixel object. It can be seen that the quality of the stored image changes when the value of BER varies. 
And finally holograms corresponding to the 500x500 pixel (solid red squares) and 800x600 pixel (empty green squares) objects were multiplexed. In these two cases, 19 and 20 holograms were stored respectively. A greater number of holograms were stored because it was possible to make better use of the dynamic range. As can be seen, the first 15 holograms have a BER of less than 0.2 and the BER of the last holograms increases but never exceeds 0.4 . Figs. $4 \mathrm{e}$ to $4 \mathrm{~h}$ show the first and last holograms of these two objects.

\section{CONCLUSION}

In this study, four different data pages with a different pixel size were multiplexed in a PVA-acrylamide photopolymer. TN-LCDs were used as a spatial light modulator to modify the object beam and store the data pages in the material. The BER of the multiplexed images was calculated to quantify the quality of the images and represented versus the number of multiplexed holograms. With a thickness of $80 \mu \mathrm{m}$ the first 12 holograms all have the same BER, whose BER is less than 0.1 , which indicates that the image obtained is of very good quality, irrespective of the number of pixels of the object. This study shows the possibilities of this photopolymer as a holographic memory.

\section{ACKNOWLEDGMENT}

This work was supported by the "Ministerio de Educación y Ciencia" (Spain) under projects FIS 2005-05881-C02-01 and FIS 2005-05881-C02-02 and by the "Generalitat Valenciana" under projects ACOMP 2007/027, ACOMP 2007/020 and GV06/172.

\section{REFERENCES}

1. A. Pu and D. Psaltis, "High-density recording in photopolymer based holographic three-dimensional disks," Appl. Opt. 35, 2389-2398 (1996).

2. M. Schnoes, B. Ihas, A. Hill, L. Dhar, D. Michaels, S. Setthachayanon, G. Schomberger, and W. L. Wilson, "Holographic storage media for practical systems," in Practical Holography XVII and Holographic Materials IX, T. H. Jeong, ed., Proc SPIE 5005, 29-37 (2003).

3. R. R. McLeod, A. J. Daiber, M. E. McDonald, T. L. Robertson, T. Slagle, S. L. Sochava, and L. Hesselink, "Microholographic optical disk data storage," Appl. Opt. 44, 3197-3207 (2005).

4. S. Gallego, M. Ortuño, C. Garcia, C. Neipp, A. Belendez, I. Pascual, "High-efficiency volume holograms recording on acrylamide and N,N'methilene-bis-acrylamide photopolymer with pulsed laser", J. Mod. Opt. 52, 1575-1584, (2005).

5. S. Gallego, M. Ortuño, C. Neipp, A. Márquez, A. Bélendez, and I. Pascual, "Characterization of polyvinyl alcohol_acrylamide holographic memories with a first-harmonic diffusion model," Appl. Opt. 44, 6205-6210 (2005).

6. Ken Y. Hsu, Shiuan Huei Lin, Yi-Nan Hsiao, "Experimental characterization of phenanthrenequinode-doped poly(methyl methacrylate) photopolymer for volume holographic storage", Opt. Eng. 42, 1390-1396, (2003).

7. M. Schnoes, B. Ihas, A. Hill, L. Dhar, D. Michaels, S. Setthachayanon, G. Schomberger, and W. L. Wilson, "Holographic storage media for practical systems," in Practical Holography XVII and Holographic Materials IX, T. H. Jeong, ed., Proc SPIE 5005, 29-37 (2003).

8. Fai H. Mok, G. W. Burr and D. Psaltis, "System metric for holographic memory systems", Opt. Lett. 21, 896-898, (1996).

9. M. Ortuño, S. Gallego, C. García, C. Neipp, A. Beléndez, I. Pascual, "Optimization of a $1 \mathrm{~mm}$ thick PVA/acrylamide recording material to obtain holographic memories: method of preparation and holographic properties", Appl. Phys. B 76, 851, (2003).

10. Lu, K. Saleh, B.E.A., "Theory and design of the liquid-crystal TV as an optical spatial phase modulator", Opt. Eng. 29, 240, (1990).

11. Yamauchi, M., Eiju, T., "Optimization of twisted-nematic liquid-crystal panels for spatial light phase modulation", Opt. Commun. 115, 19, (1995).

12. A. Marquez, C .Lemmi, I. Moreno, J.A. Davis, J. Campos, M.J.Izuel, "Quantitatice prediction of the modulation behaviour of twister-nematic liquid-crystal displays based on a simple physical model" Opt. Eng. 40, 2558, (2001). 
13. Hyun Kim, Yeon H. Lee , "Unique measurement of the parameters of a twisted-nematic liquid-crystal display", Appl. Opt. 44, 1642, (2005)

14. V. Duran, J. Lancis, E. Tajahuerce, Z. Jaroszewicz, "Cell parameter determination of a twisted-nematic liquidcrystal display by single-wavelength polarimetry", J. Appl. Phys 97, 43101, (2005).

15. H. J. Coufal, D. Psaltis, G. T. Sincerbox, Holographic Data Storage, Springer-Verlag, New Cork (2000).

16. L. Dhar, K. Curtis, M. Tackitt, M. Schilling, S. Campbell, W. Wilson, A. Hill, C. Boyd, N. Levinos, A. Harris, "Holographic storage of multiple high-capacity digital data pages in thick photopolymer systems", Opt. Lett. 23, 1710, (1998).

17. P. Varhegyi, P. Koppa, F. Ujhelyi, Lorincz, "System modeling and optimization of Fourier holographic memory" Appl. Opt. 44, 3024, (2005).

18. E. Fernández, M. Ortuño, S. Gallego, C. García, A. Beléndez, I. Pascual, “Comparison of peristrophic multiplexing and a combination of angular and peristrophic holographic multiplexing in a thick PVA/acrylamide photopolymer for data storage", Appl. Opt. 46, 5368-5373, (2007).

19. E. Fernández, M. Ortuño, A. Márquez, S. Gallego, A. Beléndez and I. Pascual, "Optimization of a holographic memory using an LCD and a PVA based photopolymer", in Liquid Crystals and Applications in Optics, M. Glogarova, P. Palffy-Muhoray and M. Copic eds., Proc. Of SPIE 6587, 65870J/1 - 65870J/9, (2007).

20. A. Márquez, J. Campos, M. J. Yzuel, I. Moreno, J. A. Davis, C. Iemmi, A. Moreno and A. Robert, "Characterization of edge effects in twisted nematic liquid crystal displays", Opt. Eng. 39, 3301-3307, (2000). 